\title{
The most common infections leading to hospitalization in educational -research hospital of Yazd
}

\author{
Jamshid Ayatollahi ${ }^{1}$, Amirhossein Azimimeybodi ${ }^{2}$, Zohreh AkhoundiMeybodi ${ }^{3}$, Seyed Hossein \\ Shahcheraghi ${ }^{*}$
}

1 Professor, Infectious Diseases Research Center, Shahid Sadoughi Hospital, Shahid Sadoughi University of Medical Sciences, Yazd, Iran 2 Medical student, Shahid Sadoughi University of Medical Sciences, Yazd, Iran

3 Infectionlogist, Fellowship of prevention and control of nosocomial infections, Shahid Sadoughi Hospital, Shahid Sadoughi University of Medical Sciences, Yazd, Iran

4 Researcher, Infectious Diseases Research Center, Shahid Sadoughi Hospital, Shahid Sadoughi University of Medical Sciences, Yazd, Iran

*Corresponding Author: Seyed Hossein Shahcheraghi, Researcher, Infectious Diseases Research Center, Shahid Sadoughi Hospital, Shahid Sadoughi University of Medical Sciences, Yazd, Iran.

E-mail: shahcheraghih@gmail.com, Tel: +98-913-2531389

\section{Abstract}

Infectious diseases are one of the diseases that are generally better suited to diagnostic and antibiotic treatments. However, there is significant mortality in infectious diseases. This study was done to determine the infection leading to admission. This study was performed on hospital data. All patients admitted in Shahid Sadoughi hospital in Yazd province in one year were studied. The final diagnosis is based on symptoms and clinical infectious disease by a specialist. Data were analyzed by statistical software SPSS and Chi-square tests. In the time period examined, 1326 patients were enrolled, $56.7 \%$ were male and $43.3 \%$ female that age range were $14-95$ years. The most commonly reported illness at the time of hospital discharge was pneumonia (16.7\%), UTI (11.2\%), diabetic foot ulcer and cellulite (13.7\%). There was a significant relationship between the most commonly diagnosed illnesses age and gender at the onset and discharge of patients ( $P$ value $<0.05)$. It is worth noting that cases without diagnosis were $20.1 \%$ and in the elderly group with a population of $53.6 \%$ of the total hospitalized patients, pneumonia and UTI were the most common complications. The present study has shown that pneumonia is the most prevalent among the causes of hospitalization among the elderly and non-elderly patients in the infectious part, and there is a significant relationship between age and sex and the prevalence of disease in patients were in Hospital.

Keywords: Infections, Admission, Pneumonia, UTI.

\section{INTRODUCTION}

Infectious diseases are among the diseases that are usually cured with appropriate diagnostic measures and antibiotic treatments ${ }^{[1]}$. However, there is also significant mortality in infectious diseases ${ }^{[2]}$. On the one hand, this mortality can be due to the acute and urgent nature of infectious diseases, so that the speed of starting treatment plays a decisive role in the prognosis of the disease, and on the other hand, in chronic infectious diseases such as tuberculosis and HIV infection. Mortality can be due to complications from the disease itself or concomitant diseases such as cardiovascular problems ${ }^{[3,4]}$. Given that acute or chronic infectious diseases are one of the main causes of death in patients in hospitals, proper follow-up and treatment of these patients is of particular importance and requires experienced staff and appropriate para-clinical measures ${ }^{[5]}$.

Pneumonia as a clinical syndrome is also one of the ten most common causes of death in people 65 and older and the most common cause of death due to infections ${ }^{[6,7]}$. About $10 \%$ of patients with pneumonia have severe disease and the mortality of patients is about $28 \%{ }^{[7]}$. Bacterial tuberculosis is also a chronic disease caused by Mycobacterium tuberculosis. Currently, tuberculosis is the deadliest adult microbial disease in the world and is expected to continue until $2020{ }^{[8]}$. 
Urinary tract infection is also one of the most common bacterial infections that are of the highest importance after respiratory tract infection [9]. In the United States, urinary tract infections cause infections in 7-8 million outpatients and more than onethird of hospital infections per year [10]. Other microorganisms that can be infected include Proteus mirabilis, various species of Klebsiella, Enterobacter and Pseudomonas [11].

Skin and soft tissue infections are important causes of outpatient visits to medical clinics or hospitals [12]. These infections have a wide range and can range from a superficial infection that requires time to heal to severe and dangerous cases that lead to death without prompt diagnosis [12]. Research shows that soft tissue infections occur 1.5 times more often in the lower extremities of diabetic patients than in other patients. Infections may cause metabolic disorders, and metabolic disorders may facilitate infection [13]. Immune factors influencing the development of infection in diabetics include neutrophil dysfunction (including endothelial adhesion, chemotaxis, and phagocytosis). Inadequate blood sugar control also increases the prevalence of infections in diabetics [14].

The aim of this study was to investigate the most common infectious agents leading to hospitalization and to identify these factors in order to improve and provide better clinical and paraclinical services to patients. Preventive decisions on infectious diseases were also selected.

\section{MATERIALS AND METHODS}

This research was applied and was performed descriptivelyanalytically on hospital data. The study population in this research were all patients who were hospitalized in the infectious ward of Shahid Sadoughi Hospital in Yazd between October 2017 and September 2018. Sampling in this study was done by census method. In this study, 1326 cases were reviewed.

Demographic and clinical information in the files of patients admitted to the infectious ward of Shahid Sadoughi Hospital were collected for one year and the cases of respiratory, skin, urogenital, gastrointestinal, etc. infections that led to hospitalization were identified and recorded.

This plan was carried out in compliance with all the principles of ethics in research approved by the Ministry of Health and was approved by the University Ethics Committee. In addition, information about patients is completely confidential and is provided to the relevant authorities if needed.

Considering that the study population included all patients admitted to the infectious ward of Shahid Sadoughi Hospital in Yazd, in order to properly study the case information of these patients, including name, surname, age, sex, cause of hospitalization and duration of hospitalization the basis of the physician's diagnosis and the presence of underlying diseases were accurately recorded. Other clinical information related to the individual's disease was also recorded with their consent.

\section{Statistical analysis}

Accurate recording of demographic information and related clinical cases in patients admitted to the infectious ward in SPSS software version 21 and chi-square tests were identified as the two most common diseases leading to hospitalization in the subjects.

\section{RESULTS}

The mean age of patients in the present study was 52.85 with a standard deviation of 20.75 and a range of changes of 14 to 95 years. Among the patients, 574 (43.3\%) were female and 752 $(56.7 \%)$ were male.

Patients were divided into age groups whose frequency distribution showed that the lowest frequency was related to the age group under 20 years $(4.5 \%)$. The highest prevalence of infectious diseases was in the 4th (18.6\%) and 7th $(16.8 \%)$ decades and approximately $46.4 \%$ of patients were under 50 years old and the rest were over 50 years old.

The most common causes of hospitalization at the time of admission were pneumonia, wound infection and cellulite and urinary tract infections, respectively, which were $15.6 \%, 12.8 \%$, $10.7 \%$ and $4.6 \%$, respectively.

The most common diagnoses at discharge were pneumonia, wound and cellulite infections, urinary tract infections and tuberculosis, respectively, which were $16.7 \%, 13.7 \%, 11.2 \%$ and $4.8 \%$, respectively.

In the study of common diseases in men and women, urinary tract infections, pneumonia, meningitis, tuberculosis were more common in women and sepsis, upper respiratory tract infections, wound and cellulite infections, hepatitis and brucellosis were more common in men. There was a significant relationship between the types of hospitalization reasons at the time of admission and the sex of the patients.

Pneumonia and urinary tract infections were the most common causes of hospitalization in all age groups except those under 20 years of age. In the 6th and 7th decades, diabetic wound infection and cellulite were more common than pneumonia, but in the 8th and 9th decades, urinary tract infections and pneumonia were more common. A significant relationship was observed between the causes of hospitalization and age groups of patients.

In the study of common diseases in men and women, among the causes of hospitalization at discharge, urinary tract infection, pneumonia and tuberculosis were more common in women and sepsis, upper respiratory tract infections, wound and cellulite infections, hepatitis and brucellosis were more common in men. There was a significant relationship between the types of hospitalization reasons at the time of discharge and the sex of the patients (Table 1). 
Table 1: Frequency distribution of the most common diseases based on gender at the time of discharge in the studied patients

\begin{tabular}{|l|l|l|l|l|l|l|}
\hline \multirow{2}{*}{ Diagnosis } & \multicolumn{2}{l}{ Woman } & \multicolumn{2}{l|}{ Man } & \multicolumn{2}{l|}{ Total } \\
\cline { 2 - 7 } & Number & Percent (\%) & Number & Percent (\%) & Number & Percent (\%) \\
\hline Pneumonia & 125 & 21.8 & 97 & 12.9 & 222 & 16.7 \\
\hline Wound infection and Cellulitis & 65 & 11.3 & 117 & 15.6 & 182 & 13.7 \\
\hline Urinary tract infection & 82 & 14.3 & 66 & 8.8 & 148 & 11.2 \\
\hline Tuberculosis & 39 & 6.8 & 25 & 3.3 & 64 & 4.8 \\
\hline Brucellosis & 22 & 3.8 & 29 & 3.9 & 51 & 3.8 \\
\hline Gastroenteritis & 21 & 3.7 & 21 & 2.8 & 42 & 3.2 \\
\hline Sepsis & 15 & 2.6 & 27 & 3.6 & 42 & 3.2 \\
\hline Upper respiratory infection & 6 & 1 & 31 & 4.1 & 37 & 2.8 \\
\hline Meningitis & 12 & 2.1 & 12 & 1.6 & 24 & 1.8 \\
\hline Hepatitis & 2 & 0.3 & 9 & 1.2 & 11 & 0.8 \\
\hline Malignancy & 5 & 0.8 & 11 & 1.4 & 16 & 1.2 \\
\hline Other & 79 & 13.8 & 141 & 18.8 & 220 & 16.7 \\
\hline No Diagnosis & 101 & 17.6 & 166 & 22.1 & 267 & 20.1 \\
\hline Total & 574 & 100 & 752 & 100 & 1326 & 100 \\
\hline p-value & 0.001 & & & & \\
\hline
\end{tabular}

In terms of the distribution of disease prevalence in different age groups, office infection was more prevalent in the 6th, 7th, 8th and higher decades. But pneumonia was most prevalent in the 4th decade and has been more common since then at older ages. Hepatitis was also more common in people under the age of 50 . Brucellosis was most prevalent in the 4th decade. The highest prevalence of tuberculosis was in the 4th and 5th decades.

\section{DISCUSSION}

Infectious diseases are among the diseases that are usually cured with appropriate diagnostic measures and antibiotic treatments [15].

However, there is also significant mortality in infectious diseases. On the one hand, this mortality can be due to the acute and urgent nature of infectious diseases, so that the speed of treatment begins to play a decisive role in the prognosis of the disease, and on the other hand, in chronic infectious diseases such as tuberculosis and HIV infection. Mortality can be due to complications from the disease itself or concomitant diseases such as cardiovascular problems ${ }^{[5]}$.

In the present study, the most common causes of hospitalization upon admission were pneumonia, wound infection and cellulite, and urinary tract infections, respectively. Also in this study and in the study of common diseases in men and women, urinary tract infections, pneumonia, meningitis, tuberculosis were more common in women and sepsis, upper respiratory tract infections, wound and cellulite infections, hepatitis and brucellosis were more common in men. There was a significant relationship between the types of hospitalization reasons at the time of admission and the sex of the patients. In the 6th and 7th decades, diabetic wound infection and cellulite were more common than pneumonia, but in the 8th and 9th decades, urinary tract infections and pneumonia were more common. A significant relationship was observed between the causes of hospitalization and age groups of patients. In 2016, Sadeghi et al. In Iran reviewed the files of 201 elderly patients admitted to the infectious ward of Imam Khomeini Hospital in Kermanshah during 2009-2012, and declared the most common infectious disease to be pneumonia with a frequency of $45 \%$, and the most common complaint of patients was fever $(48.8 \%)$ reported [16].

During a study in 2011, Ahmadi et al. Examined infections leading to hospitalization in both the elderly and non-elderly groups at Razi Hospital in Ahvaz. Tuberculosis was detected in $0.8 \%$. Also, the cases of respiratory tract infections were $2.1 \%$, sepsis was $9.6 \%$ and genitourinary tract infections were $0.9 \%$. This study reported an increased risk of infection in the elderly over the age of 60 compared to the elderly, as well as deaths in the elderly, which should be given more attention to the methods of care for such diseases in the elderly [17].

Golsha et al. In 2012, during a descriptive-analytical study conducted in Gorgan 5 Azar Hospital in 2009-2010, by referring to the files of elderly patients aged 65 years and older and examining them, it was determined that the deaths (8 cases) without a specific cause and sepsis was the second most common cause of death in these patients [18].

Heravi et al. In 2011 to evaluate elderly patients admitted to the infectious ward of Shahid Beheshti Hospital in Kashan, out of 248 patients studied, 133 (53.6\%) died, the most common complaints of patients with fever $(19.4 \%)$ and shortness of breath $(14.9 \%)$ and the most common underlying disease was hypertension (37\%) and the most common infectious disease was pneumonia (23.4\%) followed by sepsis (15.7\%) ${ }^{[19]}$.

During a study by Hasabi et al. In 2007, they investigated the causes of death due to infectious diseases in the infectious ward. During three years, 3976 people were hospitalized in Imam Khomeini Hospital in Tehran. Sepsis was reported in $22 \%$ of bacterial pneumonias and $19 \%$ of tuberculosis ${ }^{[20]}$.

During a study by Golsha et al. In 2005, referring to one-year medical records, they evaluated 122 cases of diabetic patients older than 20 years in 5 Azar Hospital in Gorgan and the reason for hospitalization of 60 people (49.3\%) Soft tissue and diabetic foot infections, $36 \%$ sepsis, $5.7 \%$ pneumonia, 3.2\% urinary tract infections and $5.7 \%$ were reported to be unknown ${ }^{[21]}$. 


\section{CONCLUSION}

In the present study, there is a significant relationship between the cases of common diseases in the infectious ward and age and gender at the time of admission and discharge, and the elderly are a group of people in the community who due to physical reasons and living conditions in They are exposed to the above-mentioned common infectious diseases with a higher percentage than young people, and clinical and paraclinical approaches and measures should be considered at the right time and accuracy for both the elderly and non-elderly groups.

\section{Acknowledgement}

The authors thank infectious diseases research center of Yazd, Iran for their contribution.

\section{Conflict of Interest}

We declare that we have no conflict of interest.

\section{Financial Support}

None declared.

\section{REFERENCES}

1. Skiest DJ, Brown K, Cooper TW, Hoffman-Roberts H, Mussa HR, Elliott AC. Prospective comparison of methicillin-susceptible and methicillin-resistant community-associated Staphylococcus aureus infections in hospitalized patients. J Infect. 2007; 54(5):427-34.

2. Bajaj JS, O'Leary JG, Reddy KR, Wong F, Olson JC, Subramanian RM, et al. Second infections independently increase mortality in hospitalized patients with cirrhosis: the North American consortium for the study of end-stage liver disease (NACSELD) experience. Hepatology. 2012; 56(6):2328-35

3. Soria J, Metcalf T, Mori N, Newby RE, Montano SM, Huaroto L, et al. Mortality in hospitalized patients with tuberculous meningitis. BMC Infect Dis. 2019; 19(1):9

4. Worodria W, Emily C, Andama A, Sanyu I, Byanyima P, Musisi E, et al. Predictors of Mortality Among Hospitalized Patients With Lower Respiratory Tract Infections in a High HIV Burden Setting. Journal of acquired immune deficiency syndromes (1999). 2018; 79(5):624.

5. Obel N, Thomsen HF, Kronborg G, Larsen CS, Hildebrandt PR, Sørensen HT, et al. Ischemic heart disease in HIV-infected and HIV-uninfected individuals: a population-based cohort study. Clin Infect Dis. 2007; 44(12):1625-31.

6. Wang D, Hu B, Hu C, Zhu F, Liu X, Zhang J, et al. Clinical characteristics of 138 hospitalized patients with 2019 novel coronavirus-infected pneumonia in Wuhan, China. JAMA. 2020; 323(11):1061-9.

7. Torres A, Sibila O, Ferrer M, Polverino E, Menendez R, Mensa J, et al. Effect of corticosteroids on treatment failure among hospitalized patients with severe community-acquired pneumonia and high inflammatory response: a randomized clinical trial. JAMA. 2015; 313(7):677-86.

8. Blanc F-X, Badje AD, Bonnet M, Gabillard D, Messou E, Muzoora $\mathrm{C}$, et al. Systematic or Test-Guided Treatment for Tuberculosis in HIV-Infected Adults. N Engl J Med. 2020; 382(25):2397-410.

9. Eliakim-Raz N, Babitch T, Shaw E, Addy I, Wiegand I, Vank C, et al. Risk factors for treatment failure and mortality among hospitalized patients with complicated urinary tract infection: a multicenter retrospective cohort study (RESCUING Study Group). Clin Infect Dis. 2019; 68(1):29-36.

10. Liangpunsakul S. Clinical characteristics and mortality of hospitalized alcoholic hepatitis patients in the United States. J Clin Gastroenterol. 2011; 45(8):714.

11. van den Hoogen BG, van Doornum GJ, Fockens JC, Cornelissen JJ, Beyer WE, Groot Rd, et al. Prevalence and clinical symptoms of human metapneumovirus infection in hospitalized patients. The Journal of infectious diseases. 2003; 188(10):1571-7.

12. Lipsky BA, Weigelt JA, Gupta V, Killian A, Peng MM. Skin, soft tissue, bone, and joint infections in hospitalized patients: epidemiology and microbiological, clinical, and economic outcomes. Infect Control Hosp Epidemiol. 2007; 28(11):1290-8.

13. Raslan M, Gonzalez MC, Dias MCG, Nascimento M, Castro M, Marques $\mathrm{P}$, et al. Comparison of nutritional risk screening tools for predicting clinical outcomes in hospitalized patients. Nutrition. 2010; 26(7-8):721-6.

14. Allard R, Leclerc P, Tremblay C, Tannenbaum T-N. Diabetes and the severity of pandemic influenza $A(\mathrm{H} 1 \mathrm{~N} 1)$ infection. Diabetes Care. 2010; 33(7):1491-3.

15. Fridkin S, Baggs J, Fagan R, Magill S, Pollack LA, Malpiedi P, et al. Vital signs: improving antibiotic use among hospitalized patients. MMWR Morbidity and mortality weekly report. 2014; 63(9):194.

16. Sadeghi S, Bilvayeh S, Afzali S, Bahmani T, Almasi A, Mahdavian B. The Most Common Causes Hospitalization of Elderly Patients In The Infectious Ward Of Imam Khomeini Hospital, Kermanshah (2010-2014). 2016. [In Persian]

17. Ahmadi F, Roozbeh F. Infections leading to admission in elderly and non-elderly groups in a referral teaching hospital in. Journal of Mazandaran University of Medical Sciences. 2013; 23(105):117-120. [In Persian]

18. Golsha R, Golshah E, Jalali Z, Rajabli N, Roshandel G. Final Diagnosis and Outcomes in Old Patients Admitted to the Infectious Diseases Ward. medical journal of mashhad university of medical sciences. 2012; 55(3):158-63. [In Persian]

19. Momen Heravi M, Afzali H, Soleimani Z, Matin M. Common infectious diseases among the hospitalized elderly patients. Iranian Journal of Ageing. 2011; 6(2):64-70. [In Persian]

20. Hasibi M, Soudbakhsh A, Abadi Z, Mehdipoor P. Mortality rate of infectious disease in relation to holidays: three year study in Imam Khomeini Hospital. Tehran University Medical Journal TUMS Publications. 2008; 65(10):50-54. [In Persian]

21. Golsha R, Bazrafshan H, Rabie M. Infectious causes of hospitalization in diabetic patients, Gorgan, Iran. Medical Laboratory Journal. 2009; 3(1):158-163. [In Persian] 